\title{
Carbone-14 et environnement global : variabilité naturelle et apports anthropiques
}

\author{
P. JEAN-BAPTISTE ${ }^{1}$, M. PATERNE ${ }^{1}$
}

RÉSUMÉ Les variations passées du carbone-14 atmosphérique peuvent être reconstruites sur les derniers 50000 ans par l'étude des différences entre âges carbone-14 et âges calendaires. Liées en premier lieu à la modulation géomagnétique du taux de production du ${ }^{14} \mathrm{C}$ par le rayonnement cosmique et aux variations d'origine climatique du stock de ${ }^{14} \mathrm{C}$ océanique, ces variations ont atteint un facteur 2 au début du paléolithique supérieur. Des variations de même amplitude ont été provoquées par les essais nucléaires militaires des années 50 et 60 . La contribution cumulée de l'industrie nucléaire depuis ses débuts jusqu'à nos jours est de l'ordre d'un centième des quantités relâchées dans l'environnement par les retombées des explosions nucléaires atmosphériques. Représentant annuellement $14 \%$ du taux de production naturel de ${ }^{14} \mathrm{C}$, cette contribution au rapport isotopique ${ }^{14} \mathrm{C}^{12} \mathrm{C}$ est actuellement contrebalancée par l'effet de dilution du ${ }^{14} \mathrm{C}$ par les émissions de $\mathrm{CO}_{2}$ fossile (effet Suess).

ABSTRACT Radiocarbon and the global environment: natural variability and the human influence.

Over the last 50,000 years, atmospheric ${ }^{14} \mathrm{C}$ changes can be reconstructed from the difference between ${ }^{14} \mathrm{C}$ and calendar ages. The two main causes responsible for the observed changes in atmospheric ${ }^{14} \mathrm{C}$ are the modulation of the ${ }^{14} \mathrm{C}$ production rate by the geomagnetic field and the modification of the carbon-14 oceanic budget by climatic changes. Atmospheric ${ }^{14} \mathrm{C}$ was higher by a factor of 2 at the onset of the upper Paleolithic. Variations of similar amplitude were recorded in the 50's and early 60's, following the atmospheric detonation of nuclear bombs. The cumulative contribution of nuclear industry from its start to now is of the order of $1 / 100$ of the bomb ${ }^{14} \mathrm{C}$ inventory. Representing annually $14 \%$ of the ${ }^{14} \mathrm{C}$ natural production rate, this contribution to the ${ }^{14} \mathrm{C}^{12} \mathrm{C}$ isotopic ratio is presently counterbalanced by the dilution effect of the fossil $\mathrm{CO}_{2}$ emissions (Suess effect).

\section{Introduction}

Le carbone-14 $\left({ }^{14} \mathrm{C}\right)$ est un isotope radioactif du carbone produit naturellement dans l'atmosphère terrestre par l'action du rayonnement cosmique sur l'azote de l'air. Dans les années 50 et le début des années 60 , des quantités notables de ${ }^{14} \mathrm{C}$ ont également été injectées dans notre environnement par les essais nucléaires

\footnotetext{
1 Laboratoire des sciences du climat et de l'environnement, UMR CEA-CNRS 1572, 91191 Gif-sur-Yvette, France.
} 
atmosphériques d'origine militaire. Le carbone-14 est également présent dans les effluents des centrales nucléaires et des installations de recyclage de leur combustible, produit par différentes réactions d'activation neutronique tant dans le circuit primaire que dans le combustible et les structures internes des réacteurs. Dans les centrales électriques, les rejets proviennent du circuit primaire, le carbone-14 du combustible étant quant à lui libéré lors des opérations de recyclage. L'impact radiologique de ces rejets est faible comparé à la dose annuelle de rayonnement reçue par l'homme du fait de la radioactivité naturelle, y compris dans l'environnement immédiat des installations nucléaires (Samson et Siclet, 2002 ; Florence et Hartmann, 2002 ; Fitamant et al., 2002). Néanmoins, du fait de la réduction notable au cours de la dernière décennie des rejets totaux de radioactivité dans l'environnement, le ${ }^{14} \mathrm{C}$ contribue désormais à hauteur de $65 \%$ environ à la dose délivrée à l'homme par le parc nucléaire français (Florence et Hartmann, 2002) et à hauteur de $26 \%$ à celle générée par les activités de recyclage des combustibles par l'établissement Cogéma de La Hague (Le Bar et Devin, 2002). Si cette augmentation de la contribution relative du carbone-14 traduit avant tout l'effort global de réduction des rejets par les exploitants nucléaires, et donc un progrès des performances environnementales dans ce secteur industriel, elle explique cependant pourquoi la réglementation prévoit désormais une comptabilisation spécifique des rejets de ${ }^{14} \mathrm{C}$.

Avec une demie-vie du ${ }^{14} \mathrm{C}$ de 5730 ans, ces rejets chroniques renvoient à la question de l'accumulation à long terme du ${ }^{14} \mathrm{C}$ dans l'environnement et de son impact radiologique. Comme pour tous les radionucléides présents naturellement dans l'environnement en quantité significative, un élément d'appréciation peut être fourni par la comparaison entre la contribution liée à l'activité humaine et la contribution naturelle (actuelle ou passée).

L'objectif de cet article est de résumer notre connaissance de la variabilité naturelle du carbone-14 dans notre environnement à l'échelle du quaternaire récent (50 000 ans), et de la mettre en perspective avec la composante moderne liée à l'activité humaine.

\section{Historique de la teneur atmosphérique en carbone-14}

Depuis sa découverte par Libby en 1947 (qui lui valut le prix Nobel en 1960), le carbone-14 est devenu un outil de datation irremplaçable tant pour les sciences de la Terre que pour l'archéologie. La datation par le carbone-14 est applicable à des échantillons organiques (restes biologiques divers) ou inorganiques (eaux marines, eaux souterraines, concrétions calcaires, etc.). Dans tous les cas, l'événement daté est la rupture de l'équilibre isotopique entre l'échantillon et l'atmosphère (plongée des eaux océaniques, isolement des eaux de recharge des nappes souterraines, mort 


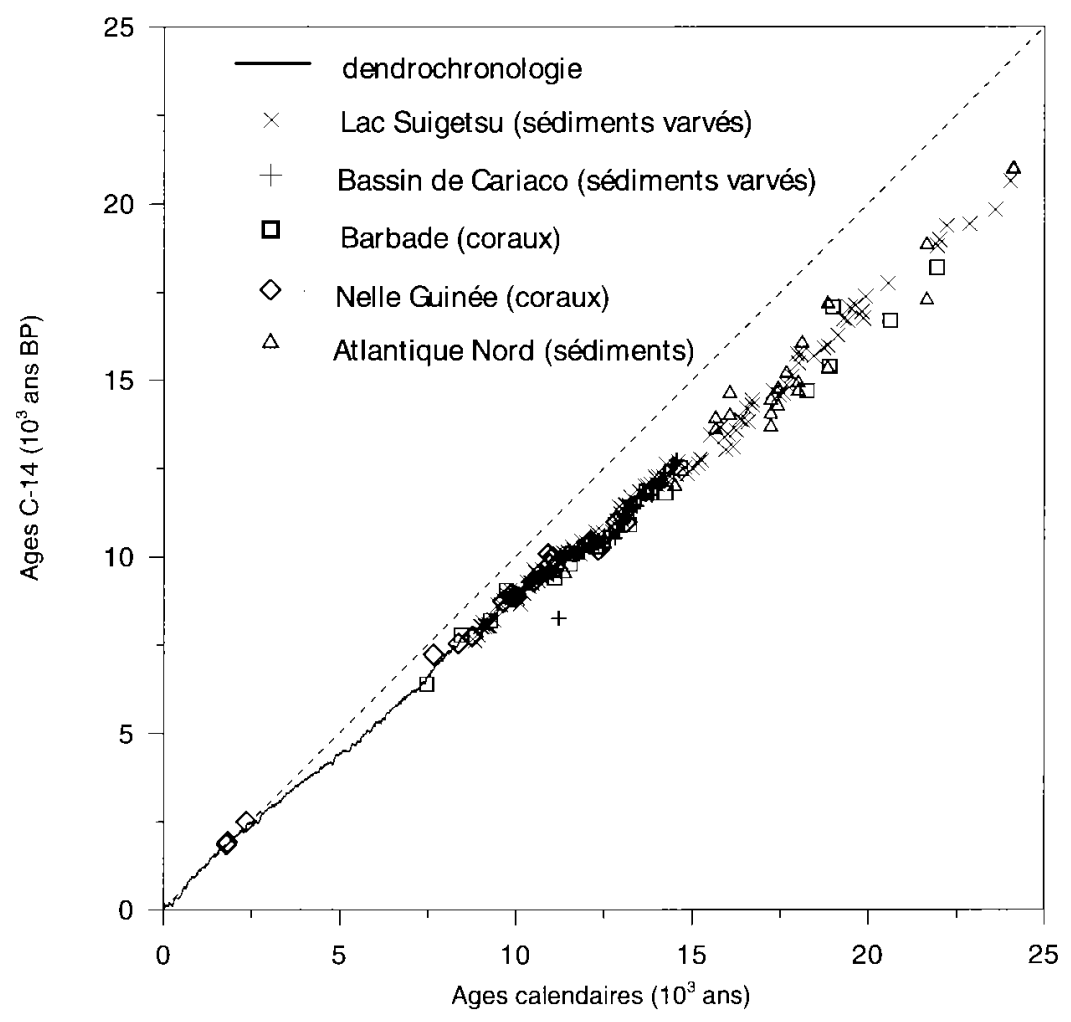

Figure 1 - Courbe de calibrage des âges ${ }^{14} \mathrm{C}$ entre l'actuel et 25000 ans BP.

${ }^{14} \mathrm{C}$ ages calibration curve back to $25,000 \mathrm{BP}$.

des organismes vivants, ...). À partir de cet événement, le rapport isotopique ${ }^{14} \mathrm{C} /{ }^{12} \mathrm{C}$ de l'échantillon décroît au rythme de la période radioactive du ${ }^{14} \mathrm{C}$. Si l'on suppose que le rapport ${ }^{14} \mathrm{C}^{12} \mathrm{C}$ atmosphérique n'a pas varié dans le passé, une datation se ramène donc à la mesure d'un rapport isotopique ${ }^{14} \mathrm{C} /{ }^{12} \mathrm{C}$. Très rapidement cependant, la découverte de différences entre l'âge ${ }^{14} \mathrm{C}$ et l'âge calendaire de différents objets archéologiques d'âges connus comme par exemple certaines momies égyptiennes, a conduit à remettre en cause l'hypothèse initiale de Libby selon laquelle la teneur naturelle en carbone-14 dans l'atmosphère et la biosphère était constante dans le temps. L'utilisation du carbone-14 pour la datation a donc nécessité de construire une courbe de calibrage reliant les âges ${ }^{14} \mathrm{C}$ aux âges calendaires (Fig. 1). Les résultats les plus nombreux et les plus précis ont été fournis par la dendrochronologie, méthode qui consiste à compter les cernes d'arbres (un cerne correspond à une année) et à comparer cet âge calendaire et 
l'âge ${ }^{14} \mathrm{C}$. Au-delà de 12000 ans $\mathrm{BP}$ (Before Present), vu la rareté des spécimens disponibles, la courbe de calibrage a dû être prolongée par d'autres moyens. L'étude des coraux fossiles, datables par le ${ }^{14} \mathrm{C}$ et également, de manière absolue, par la méthode du déséquilibre ${ }^{234} \mathrm{U} /{ }^{230} \mathrm{Th}$ (Bard et al., 1990), a permis de prolonger le calibrage avec une précision satisfaisante jusqu'à environ 20000 ans BP. Au-delà de cette période, date du dernier maximum glaciaire, les échantillons se font rares et les résultats sont actuellement en nombre insuffisant pour définir une véritable courbe de calibrage. D'autres supports physiques peuvent être utilisés, comme les stalagmites, datables par U-Th (Beck et al., 2001) ou la matière organique des sédiments varvés lacustres, datables par le comptage des varves annuelles (Kitagawa et van der Plicht, 2000). Néanmoins ces dernières méthodes reposent sur des hypothèses fragiles et difficilement vérifiables comme par exemple l'invariance des régimes de dissolution/précipitation des carbonates pour les stalagmites, en dépit des changements hydrogéologiques, ou encore la continuité temporelle de la formation des varves lacustres, quels que soient les changements climatiques. Le fait que les résultats obtenus pour les périodes les plus reculées soient en désaccord les uns avec les autres montre que des progrès sont nécessaires pour obtenir une courbe complète jusqu'à 50000 ans BP, limite pratique d'utilisation du ${ }^{14} \mathrm{C}$.

La comparaison entre l'âge calendaire (âge ${ }_{\mathrm{vraj}}$ ) et l'âge carbone-14 (âge ${ }_{\mathrm{Cl4}}$ ) donne directement accès au rapport isotopique atmosphérique $\left({ }^{14} \mathrm{C} /{ }^{12} \mathrm{C}\right) \mathrm{de}$ l'époque considérée, selon la formule :

$$
\left({ }^{14} \mathrm{C} /{ }^{12} \mathrm{C}\right)=\left({ }^{14} \mathrm{C} /{ }^{12} \mathrm{C}\right)_{1950} \exp \left[\lambda\left(\hat{a ̂ g e}_{\mathrm{vrai}}-\hat{a} g e_{\mathrm{C} 14}\right)\right]
$$

où $\left({ }^{14} \mathrm{C} /{ }^{12} \mathrm{C}\right)_{1950}$ est le rapport isotopique pré-industriel, pris conventionnellement en 1950 et égal à $1,22 \times 10^{-12}$ et $\lambda$ la constante radioactive du ${ }^{14} \mathrm{C}$.

La formule (1) ci-dessus permet donc de reconstruire l'historique de la teneur atmosphérique en carbone-14 (Fig. 2). On observe des variations notables du rapport ${ }^{14} \mathrm{C} /{ }^{12} \mathrm{C}$ atmosphérique au cours du temps, dont les deux causes principales sont :

- les changements de taux de production du carbone-14 par le rayonnement cosmique,

- la variabilité des échanges de carbone entre l'océan, l'atmosphère et la biosphère en fonction des époques climatiques.

\subsection{Variation du taux de production du carbone-14 cosmogénique}

Le taux actuel de production du carbone- 14 peut-être déterminé avec une bonne précision soit en modélisant de manière détaillée l'ensemble des réactions 
CARBONE-14 ET ENVIRONNEMENT GLOBAL

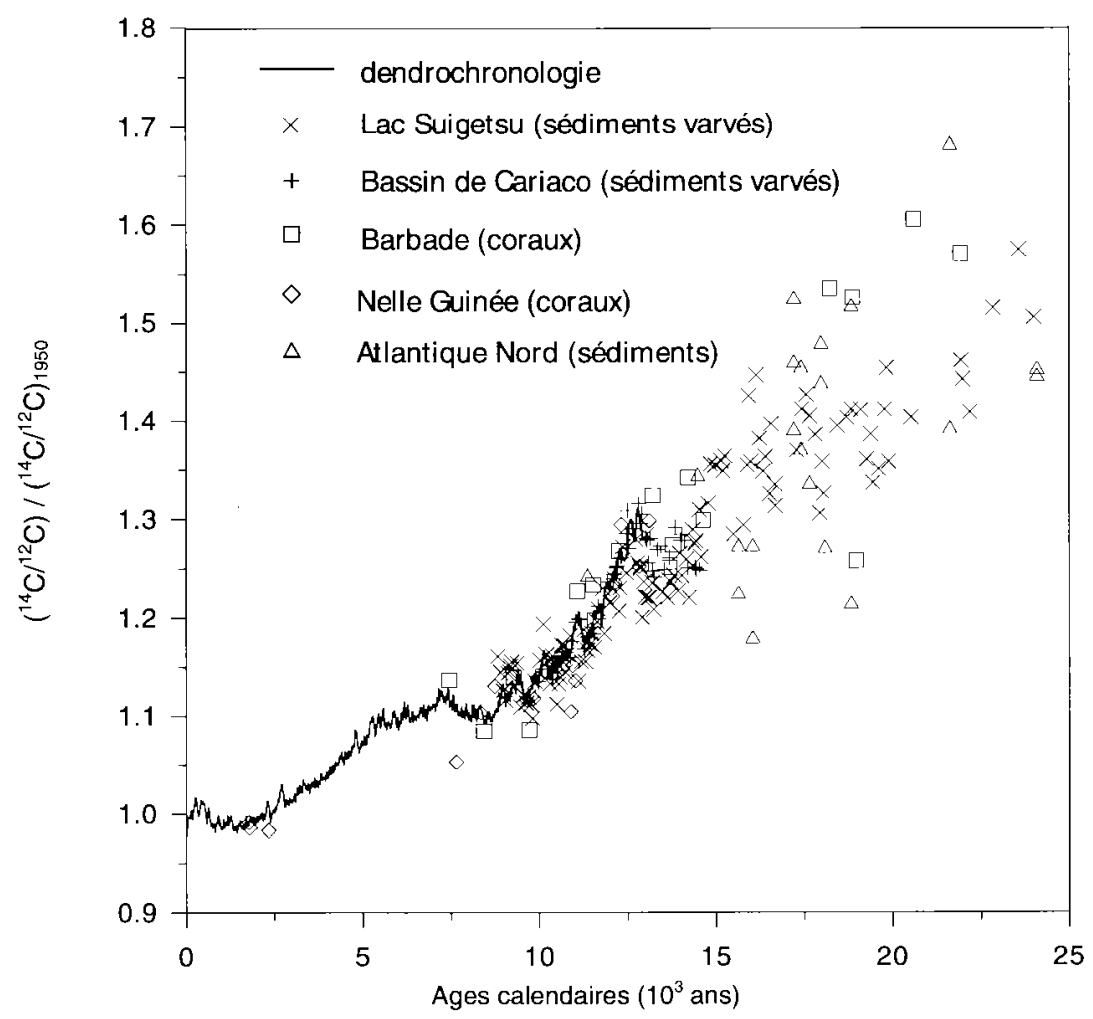

Figure 2 - Variations du rapport ${ }^{14} \mathrm{C}^{\prime 2} \mathrm{C}$ atmosphérique déduites de la courbe de calibrage $\mathrm{du}^{14} \mathrm{C}$. Variations in atmospheric ${ }^{14} \mathrm{C} /{ }^{12} \mathrm{C}$ ratio based on the ${ }^{14} \mathrm{C}$ calibration curve.

nucléaires entre le rayonnement primaire incident et les atomes de l'atmosphère terrestre (O'Brien, 1979 ; Masarik et Beer, 1999), soit en considérant que le stock de carbone-14 terrestre est à l'état stationnaire (Damon et al., 1978; Stuiver et Quay, 1980 ; Lassey et al., 1996), c'est-à-dire en supposant que le taux de production est égal au taux de disparition par décroissance radioactive. Les deux approches conduisent à un résultat cohérent de $470 \pm 70$ moles ${ }^{14} \mathrm{C} / \mathrm{an}$ (soit $1080 \pm 160 \mathrm{TBq} / \mathrm{an})$. Ce taux de production est modulé dans le temps par les variations de plusieurs paramètres :

- l'intensité du champ magnétique terrestre: le champ géomagnétique agit comme un bouclier contre les particules chargées en provenance de notre galaxie (protons et particules alpha principalement). Si son intensité augmente, le taux de production du ${ }^{14} \mathrm{C}$ diminue, et vice versa. Quand le champ 


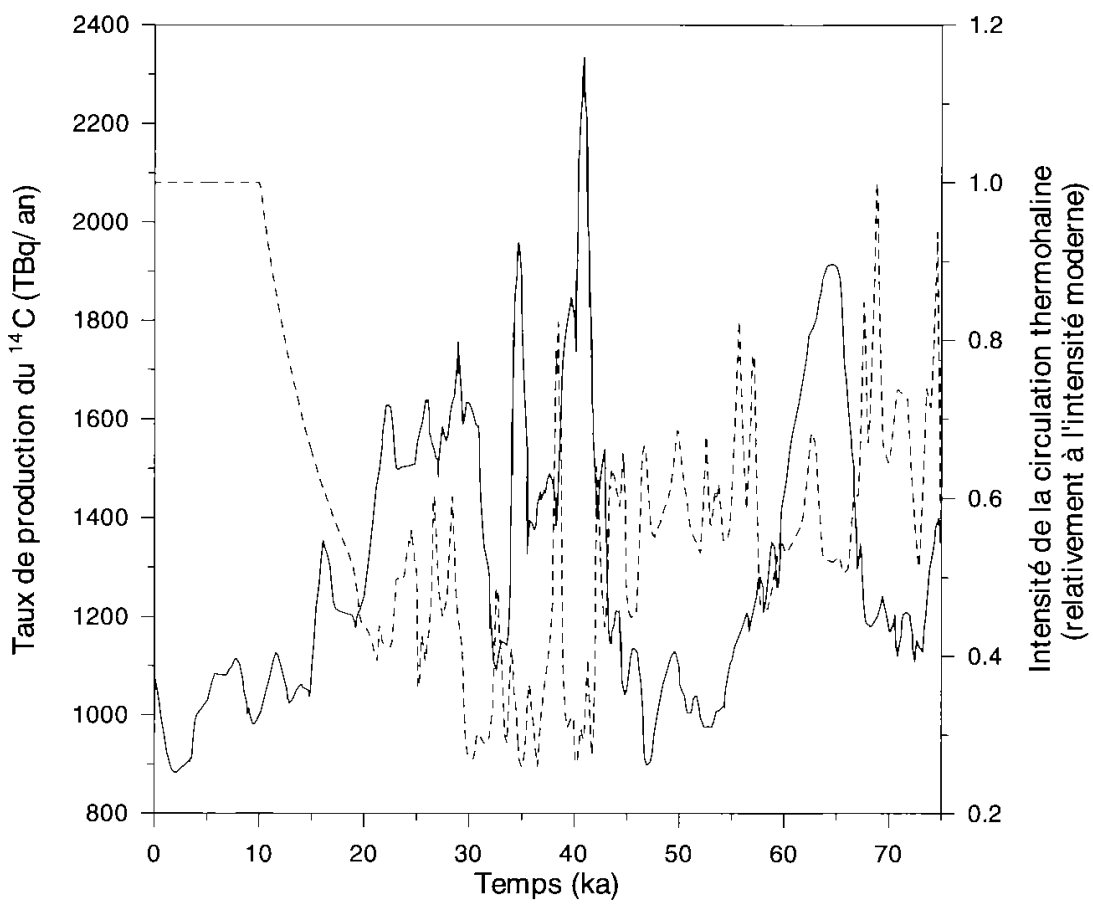

Figure 3 - (a) Variation du taux de production du carbone-14 liée aux fluctuations du champ magnétique terrestre (courbe pleine). (b) Variation relative de l'intensité de la circulation océanique thermohaline (courbe en pointillés) par rapport à l'intensité actuelle (de l'ordre de $25 \times$ $10^{6} \mathrm{~m}^{3} / \mathrm{s}$ ) - d'après Laj et al. (2002).

(a) Production rate of cosmogenic ${ }^{14} \mathrm{C} \mathrm{vs}$ time deduced from the variations in the earth magnetic field intensity (solid curve). (b) Temporal evolution of the thermohaline circulation (dashed line) relative to its present day intensity $\left(\approx 25 \times 10^{6} \mathrm{~m}^{3} / \mathrm{s}\right)-$ after Laj et al (2002).

magnétique s'annule, ce qui se produit en particulier lors d'une inversion de polarité, le taux de production est maximum. Le dernier événement connu, qui semble correspondre à une inversion de polarité avortée (excursion magnétique), est l'événement du Laschamp, daté à 40000 ans BP (Laj et al., 2000). À cette époque, qui correspond d'un point de vue de l'histoire humaine au début du paléolithique supérieur, le taux de production du ${ }^{14} \mathrm{C}$ était environ le double du taux actuel (Fig. 3a);

- l'activité solaire : les vents solaires créent également un champ électromagnétique qui s'oppose à la pénétration du rayonnement galactique dans l'héliosphère. En période de faible activité solaire, comme par exemple lors du minimum de Maunder, qui coïncide avec le petit age glaciaire, on observe que 
le taux de production du ${ }^{14} \mathrm{C}$ et des autres cosmonucléides tels le béryllium-10, augmente d'environ $25 \%$ (Bard et al., 2000);

- l'intensité du rayonnement galactique, qui peut être affectée par des événements comme par exemple l'explosion de supernovæ (Sonett et al., 1987).

Pour la période contemporaine, l'ensemble de ces paramètres est connu avec une bonne précision, en particulier grâce aux données fournies par les satellites. Dans le passé, l'activité solaire peut être reconstruite en se basant sur les observations systématiques des tâches solaires par les astronomes à partir du XVII ${ }^{\mathrm{e}}$ siècle. Pour les périodes plus anciennes, seules les variations du champ magnétique peuvent être reconstruites, à partir de la mesure de l'aimantation résiduelle des laves volcaniques ou des sédiments.

\subsection{Variabilité du cycle du carbone terrestre}

Le carbone-14 créé dans la haute atmosphère entre rapidement dans le cycle naturel du carbone sous forme de ${ }^{14} \mathrm{CO}_{2}$ et se répartit entre l'atmosphère, l'océan, et la biosphère continentale, qui sont les trois principaux réservoirs terrestres échangeant du carbone sur des échelles de temps courtes. Le tableau I montre que

\section{TABLEAU I}

Stocks pré-industriels de carbone et de ${ }^{14} \mathrm{C}$ dans les grands réservoirs superficiels. Production naturelle et apports anthropiques de ${ }^{14} \mathrm{C}$. (1) Siegenthaler et Sarmiento, 1993 ; IPCC, 2000.

(2) Lassey et al., 1996. (3) UNSCEAR, 2000. (4) Damon et al., 1978 ; O'Brien, 1979 ; Stuiver et Quay, 1980 ; Lassey et al., 1996 ; Masarik et Beer, 1999.

Pre-industrial carbon and radiocarbon stocks in the main terrestrial reservoirs. Natural production and anthropogenic releases of ${ }^{14} \mathrm{C}$.

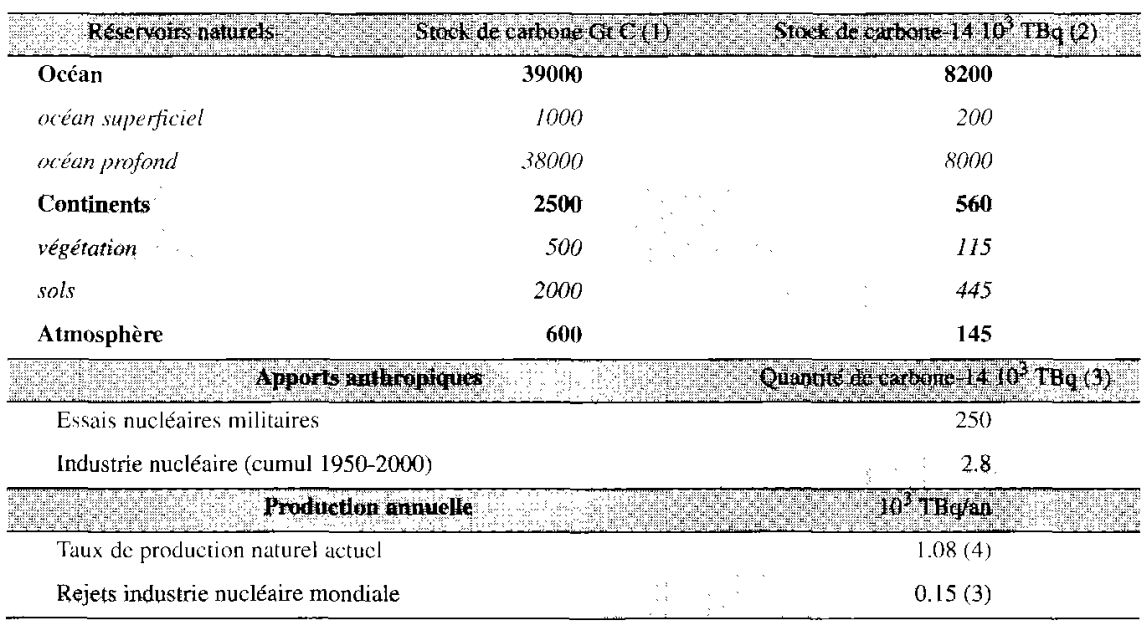


l'océan est le principal réservoir de carbone (et également de ${ }^{14} \mathrm{C}$ ). De manière très simplifiée, celui-ci peut être subdivisé en deux grands sous-ensembles :

- la couche mélangée de surface, de quelques centaines de mètres de profondeur, directement soumise à l'effet mécanique des vents et aux échanges saisonniers de chaleur. Ce réservoir de carbone s'équilibre avec l'atmosphère sur une échelle de temps de 1 à 10 ans (Broecker et Peng, 1982);

- l'océan profond, relativement isolé de l'atmosphère, et qui communique avec l'océan superficiel par des mouvements verticaux de convection. Ces mouvements de grande ampleur sont liés à l'existence de gradients de densité entre les hautes et les basses latitudes (circulation thermohaline). La mise à l'équilibre de l'océan profond s'effectue avec une constante de temps de l'ordre du millénaire, comme le montre l'analyse de sa teneur en ${ }^{14} \mathrm{C}$ (Broecker et Peng, 1982).

De manière analogue, le réservoir biosphérique de carbone possède plusieurs sousensembles dont les temps caractéristiques de mise à l'équilibre varient également entre 1 et 50 ans pour la biomasse végétale et l'humus, et plusieurs centaines d'années pour la partie réfractaire du carbone enfoui dans le sol (Trumbore et al., 1996 ; Balesdent et Recous, 1997 ; Trumbore, 2000).

La principale cause de modification dans la répartition du stock de ${ }^{14} \mathrm{C}$ entre ces trois réservoirs est liée aux changements d'intensité de la circulation thermohaline. Si son intensité augmente, le ${ }^{14} \mathrm{C}$ créé dans l'atmosphère sera transféré plus rapidement vers les grandes profondeurs, tandis que dans le cas contraire, il aura tendance à s'accumuler dans l'atmosphère.

La circulation thermohaline est relativement sensible aux conditions climatiques qui influencent la température et salinité (et donc la densité) des eaux de surface des hautes latitudes, là où se forment les eaux denses qui coulent et envahissent les fonds océaniques (Broecker, 1997). Son intensité passée peut être reconstruite à partir de différents proxys paléocéanographiques (Henderson, 2002 ; Rahmstorf, 2002). En périodes glaciaires, cette intensité est en général plus faible, comme l'atteste l'accroissement de l'écart d'âge ${ }^{14} \mathrm{C}$ entre les foraminifères planctoniques et benthiques (Shackleton et al., 1988). Elle est également plus variable. Les paléocéanographes ont ainsi montré que suite à des débâcles massives d'icebergs dans l'Atlantique-Nord (appelées évènements de Heinrich), et donc d'un apport important d'eau douce (moins dense), la circulation thermohaline pouvait se ralentir très fortement jusqu'à s'arrêter totalement, pendant plusieurs millénaires (Fig. 3b).

Sur le long terme, le cycle du carbone-14 peut être modélisé de manière très schématique par un réservoir atmosphérique et deux réservoirs océaniques dont un 


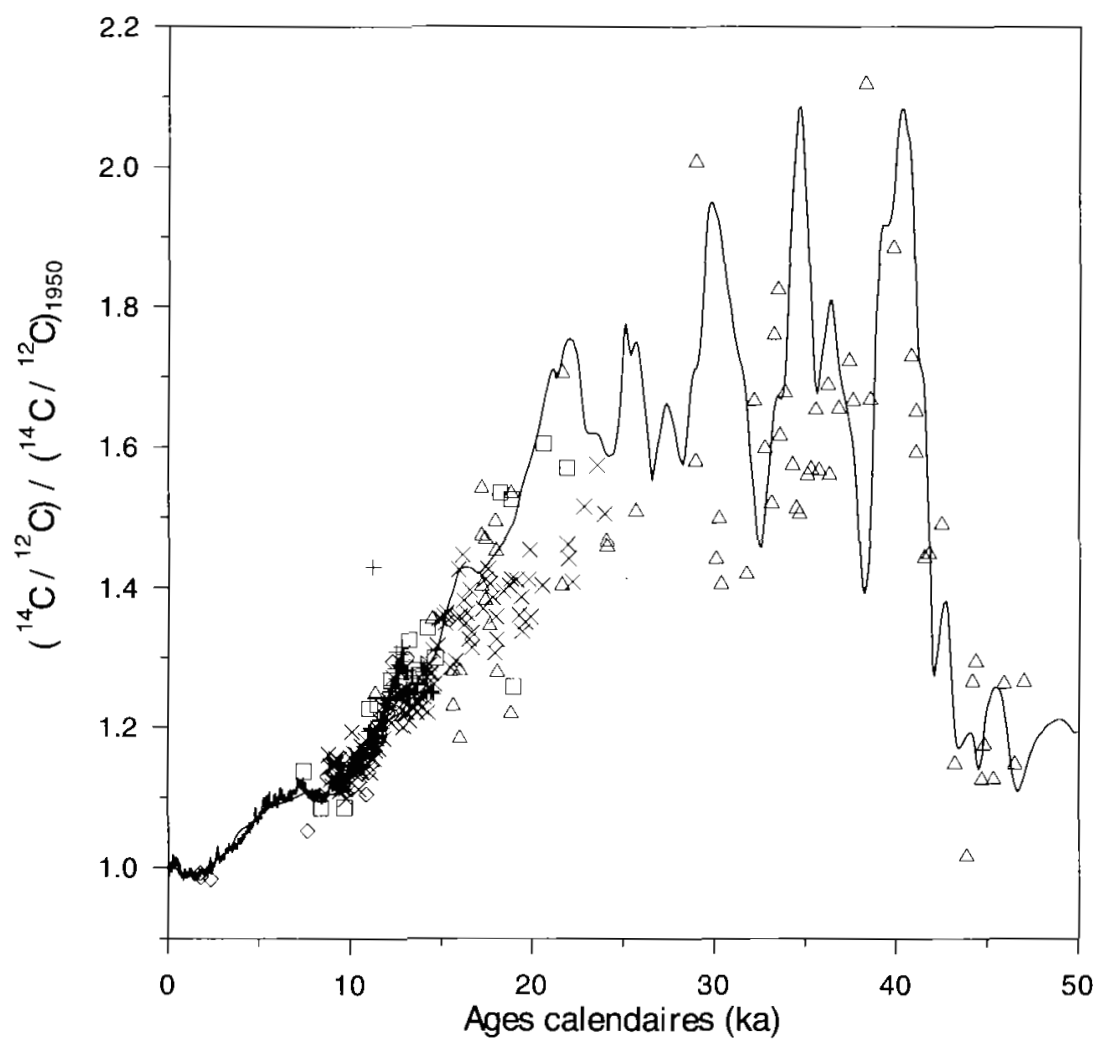

Figure 4-Simulation de l'évolution temporelle (période 0-50000 ans BP) du rapport ${ }^{14} C^{12} C$ atmosphérique avec un modèle à trois réservoirs (atmosphère, océan de surface, océan profond). Les symboles sont identiques à ceux des figures 1 et 2. Les données au-delà de 25000 ans proviennent de carottes marines de l'Atlantique-Nord (Voelker et al., 1998).

Temporal evolution of the atmospheric ${ }^{14} \mathrm{C} / 2 \mathrm{C}$ ratio (over the period 0-50,000 BP) sinulated by a three-box carbon cycle model (atmosphere, surface ocean, deep ocean). The symbols are identical to those in Figures 1 and 2. Data beyond 25,000 years come from the north atlantic cores of Voelker et aL, 1998.

réservoir superficiel et un réservoir profond (Broecker et Peng, 1982). En tenant compte des variations du taux de production de ${ }^{14} \mathrm{C}$ et de l'intensité de la circulation thermohaline (Fig. 3), ce modèle permet de décrire de manière satisfaisante la période $0-15000$ ans BP pour laquelle les données sont nombreuses et cohérentes (Fig. 4). Pour les périodes plus anciennes, la reconstruction de l'intensité de la circulation thermohaline est relativement imprécise, de même que les données concernant la valeur du rapport ${ }^{14} \mathrm{C} /{ }^{12} \mathrm{C}$ 
atmosphérique. Tout semble indiquer cependant que les fluctuations importantes (d'un facteur 2) de la teneur atmosphérique en ${ }^{14} \mathrm{C}$ observées dans les données sont dans une large mesure imputables à l'effet conjugué de la variabilité du champ magnétique et de la circulation thermohaline (Fig. 4).

\section{Comparaison des variations $d u^{14} \mathrm{C}$ atmosphérique d'origines naturelles et anthropiques}

La reconstitution des teneurs passées en carbone-14 dans l'atmosphère montre que l'humanité a connu, à certaines époques de son histoire, un environnement significativement enrichi en carbone-14 par rapport aux teneurs pré-industrielles. De telles augmentations d'origine naturelle ne sont pas à exclure dans le futur, en fonction des fluctuations du champ magnétique terrestre (Olson, 2002) et des perturbations possibles de la circulation thermohaline par le réchauffement climatique (Stocker et Schmittner, 1997).

Le fait le plus marquant de la période anthropique récente est sans aucun doute le doublement de la teneur atmosphérique en carbone-14 du fait des essais nucléaires militaires (Fig. 5). Sur la période 1950-63 qui précède le traité d'interdiction des essais atmosphériques, $(250 \pm 30) \times 10^{3} \mathrm{TBq}$ de ${ }^{14} \mathrm{C}$ ont été injectés dans l'atmosphère par les explosions d'engins nucléaires (Jain et al., 1997 ; UNSCEAR, 2000). Depuis lors, on observe une diminution substantielle du rapport ${ }^{14} \mathrm{C} /{ }^{12} \mathrm{C}$ atmosphérique par dilution progressive du ${ }^{14} \mathrm{C}$ principalement dans l'océan.

L'industrie nucléaire rejette également, dans une moindre mesure, du carbone14 dans l'environnement. En 1997, l'ensemble des rejets cumulés de ${ }^{14} \mathrm{C}$ s'élevait à $2,8 \times 10^{3} \mathrm{TBq}$ (UNSCEAR, 2000). Au niveau mondial, les rejets de ${ }^{14} \mathrm{C}$ des centrales représentent environ $11 \%$ de la production naturelle (UNSCEAR, 2000). Ceux-ci sont rapidement dilués, le marquage de l'environnement se limitant en règle générale à un rayon de quelques kilomètres autour des installations. Les effluents liquides et gazeux des opérations de recyclage du combustible nucléaire (usines de Sellafield et La Hague principalement) contiennent également du carbone-14, conduisant à un marquage régional principalement du milieu marin (Cook et al., 1998 ; Wolstenholme et al., 1998 ; Douville et al., 2002). Ces rejets sont actuellement de l'ordre de $30 \mathrm{TBq} / \mathrm{an}$, soit quelque $3 \%$ de la production naturelle. Il faut noter cependant que moins de $10 \%$ du combustible est actuellement retraité dans le monde. Dans l'hypothèse d'un recyclage généralisé, la contribution de l'industrie nucléaire s'établirait aux alentours de $30 \%$ de la production naturelle de ${ }^{14} \mathrm{C}$, avec au demeurant un impact radiologique global faible de l'ordre de $4 \mu \mathrm{Sv} / \mathrm{an}$ (UNSCEAR, 2000) en comparaison des $2400 \mu \mathrm{Sv} / \mathrm{an}$ générés en moyenne par la radioactivité naturelle. 


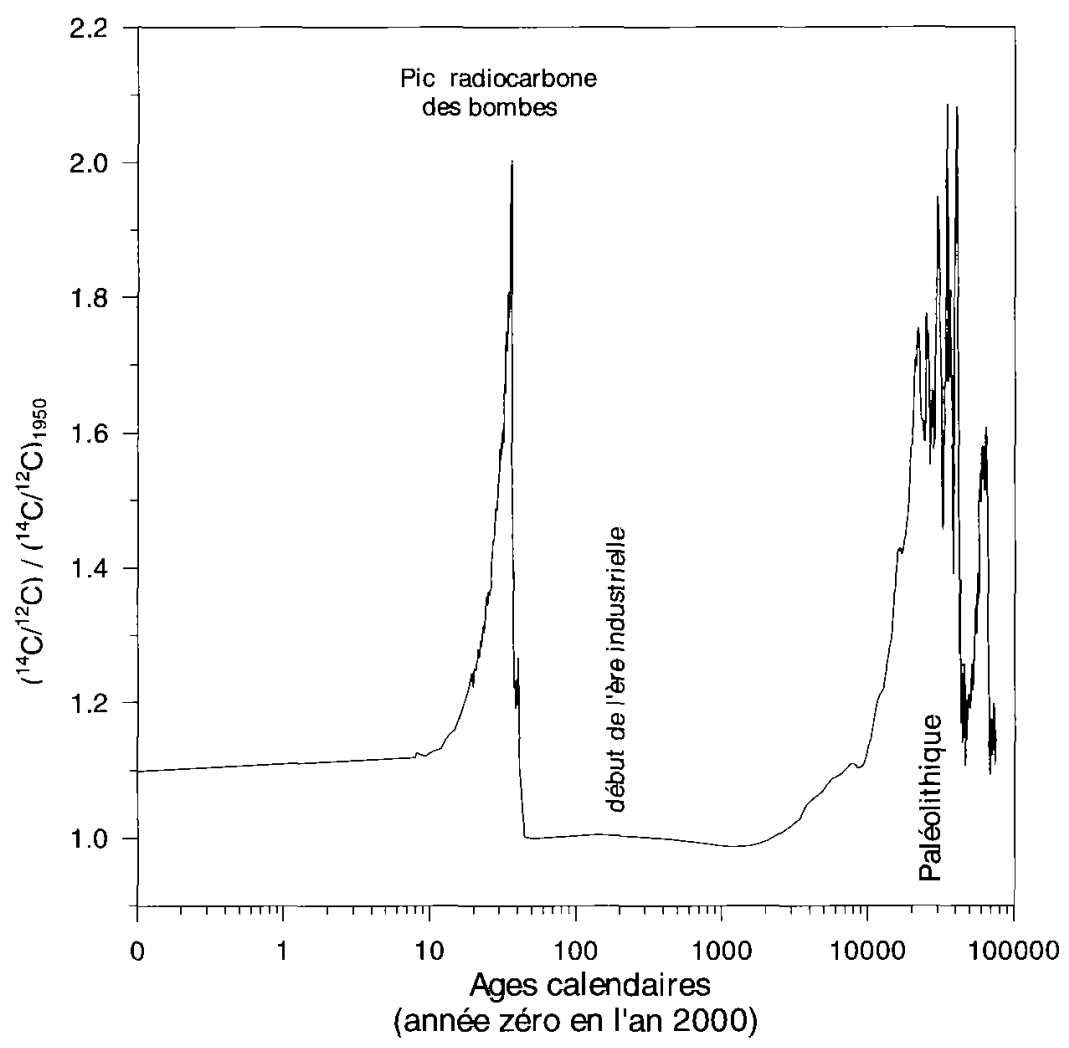

Figure 5 - Pic du carbone-14 des essais militaires atmosphériques, mis en perspective avec la variabilité naturelle du ${ }^{14} \mathrm{C}$ au quaternaire récent.

Bomb radiocarbon peak and quaternary ${ }^{14} \mathrm{C}$ natural variability.

Un dernier aspect de la période anthropique est l'effet de dilution du carbone14 par les rejets de $\mathrm{CO}_{2}$ (sans ${ }^{14} \mathrm{C}$ ) provenant de l'utilisation massive des combustibles fossiles (charbon, gaz, pétrole), connu sous le terme d'effet Suess. Sur la période industrielle 1850-1950 précédant le début des essais nucléaires militaires, 1 'analyse ${ }^{14} \mathrm{C}$ des cernes d' arbre fait ainsi apparaître une diminution de l'activité atmosphérique en ${ }^{14} \mathrm{C}$ de 2 à $3 \%$. Actuellement, six gigatonnes de carbone dénué de ${ }^{14} \mathrm{C}$ sont injectées annuellement dans l'atmosphère par les combustibles fossiles. Les prévisions de rejets, si rien n'est fait pour les limiter, dépasseront les douze milliards de tonnes/an en 2050 (scénario IS92a «Business As Usual » de l'IPCC - International Panel on Climate Change), conduisant à un doublement de la teneur atmosphérique en $\mathrm{CO}_{2}$ avant la fin du siècle par rapport à 
la teneur pré-industrielle de $280 \mathrm{ppm}$. Dans cette hypothèse, l'effet Suess se traduira en 2100 par une réduction de l'ordre de $20 \%$ du rapport ${ }^{14} \mathrm{C} /{ }^{12} \mathrm{C}$ atmosphérique (Caldeira et al., 1998), c'est-à-dire d'un ordre de grandeur équivalent à l'augmentation imputable aux rejets du secteur électronucléaire.

\section{Conclusion}

Le rapport ${ }^{14} \mathrm{C} /{ }^{12} \mathrm{C}$ atmosphérique est sujet à des variations importantes d'origine naturelle, liées à différents phénomènes géophysiques et climatiques. Pendant l'événement magnétique du Laschamp (40 000 ans BP) correspondant au début du paléolithique supérieur, la teneur atmosphérique en ${ }^{14} \mathrm{C}$ était deux fois plus élevée qu'en 1950. Les variations de la circulation océanique thermohaline, modulées par les changements climatiques, jouent également un rôle important dans la répartition du carbone-14 entre l'océan et l'atmosphère. À plus court terme, le taux de production du carbone-14 est en outre modulé, dans une certaine mesure, par les propriétés magnétiques des vents solaires.

La période anthropique a été marquée par le pic de radiocarbone des essais nucléaires militaires, qui ont à nouveau fait doubler la teneur atmosphérique en ${ }^{14} \mathrm{C}$ au début des années 60.

Au niveau actuel de puissance installée, les rejets de ${ }^{14} \mathrm{C}$ du secteur électronucléaire représentent $14 \%$ du taux de production naturel. L'augmentation du rapport isotopique ${ }^{14} \mathrm{C} /{ }^{12} \mathrm{C}$ liée à cette contribution est contrebalancée par 1'effet de dilution (effet Suess) du ${ }^{14} \mathrm{C}$ par les émissions massives de carbone d'origine fossile.

\section{RÉFÉRENCES}

Balesdent J., Recous S. (1997) The residence times of C, and the potential for C storage in some French cultivated soils, Can. J. Soil Sci. 77, 187-193.

Bard E., Hamelin B., Fairbanks R.G., Zindler A. (1990) Calibration of ${ }^{14} \mathrm{C}$ timescale over the past 30,000 years using mass-spectrometric U-Th ages from Barbados corals, Nature 345, 405-410.

Bard E., Raisbeck G., Yiou F., Jouzel J. (2000) Solar irradiance during the last 1200 years based on cosmogenic nuclides, Tellus B 52, 985-992.

Beck J.W., Richards D.A., Edwards R.L., Silverman B.W., Smart P.L., Donahue D.J., HererraOsterheld S., Burr G.S., Calsoyas L., Jull A.J., Biddulph D. (2001) Extremely large variations of atmospheric ${ }^{14} \mathrm{C}$ concentration during the last glacial period, Science 292, 2453-2458.

Broecker W.S. (1997) Thermohaline circulation, the Achilles heel of our climate system: will manmade $\mathrm{CO}_{2}$ upset the current balance? Science 278, 1582-1588.

Broecker W.S., Peng T.H. (1982) Rates of vertical mixing and sediment accumulation, Tracers in the sea, Chapter 5, pp. 236-274. Lamont-Doherty Geological Observatory, Columbia University, Eldigio Press, New York, $690 \mathrm{pp}$. 


\section{CARBONE-14 ET ENVIRONNEMENT GLOBAL}

Caldeira K., Rau G.H., Duffy P.B. (1998) Predicted net efflux of radiocarbon from the ocean and increase in atmospheric radiocarbon content, Geophys. Res. Lett. 25, 381 1-3814.

Cook G.T., MacKenzie A.B., Naysmith P., Anderson R. (1998) Natural and anthropogenic ${ }^{14} \mathrm{C}$ in the UK coastal marine environment, J. Environ. Radioactivity 40, 89-111.

Damon P.E., Lerman J.C., Long A. (1978) Temporal fluctuations of the atmospheric ${ }^{14} \mathrm{C}$ : causal factors and applications, Ann. Rev. Earth Planet. Sci. 6, 457-494.

Douville E., Fievet B., Germain P., Fournier M. (2002) Comportement du carbone-14 dans le milieu marin à proximité de l'usine de Cogéma-La Hague (Goury)-France, Actes de la journée carbone-14 de la SFRP (18 avril 2002), Société française de radioprotection, Paris.

Fitamant M.L., Piccione M., Dellero N. (2002) Les rejets de carbone-14 et la surveillance de l'environnement de l'établissement Cogéma-La Hague, Actes de la journée carbone-14 de la SFRP (18 avril 2002), Société française de radioprotection, Paris.

Florence D., Hartmann P. (2002) Les rejets radioactifs des centrales nucléaires et leur impact radiologique, Actes du Colloque de la Société française de radioprotection "Evaluation et surveillance des rejets radioactifs des installations nucléaires ", Strasbourg, 13-14 novembre 2002.

Henderson G.M. (2002) New oceanic proxies for paleoclimate, Earth Planet. Sci. Lett. 203, 1-13.

IPCC (2000) Land use, Land-use change and forestry. Intergovernmental Panel on Climate Change special report. Cambridge University Press, UK, pp. 377.

Jain A.K., Kheshgi H.S., Wuebbles D.J. (1997) Is there an imbalance in the global budget of bombproduced radiocarbon? J. Geophys. Res. 102, 1327-1333.

Kitagawa H., van der Plicht J. (2000) Atmospheric radiocarbon calibration beyond 11,900 cal BP from lake Suigetsu laminated sediments, Radiocarbon 42, 369-380.

Laj C., Kissel C., Mazaud A., Channell J.E., Beer J. (2000) North Atlantic paleointensity stack since $75 \mathrm{ka}$ (NAPIS-75) and the duration of the Laschamp event, Phil. Trans. R. Soc. Lond. A 358, 1009-1025.

Laj C., Kissel C., Mazaud A., Michel E., Muscheler R., Beer J. (2002) Geomagnetic field intensity, North Atlantic deep water circulation and atmospheric ${ }^{14} \mathrm{C}$ during the last $50 \mathrm{kyr}$, Earth Planet. Sci. Lett. 200, 177-190.

Lassey K.R., Enting I.G., Trudinger C.M. (1996) The earth's radiocarbon budget, Tellus B 48, 487-501.

Le Bar S., Devin P. (2002) Les rejets radioactifs du site de Cogéma-La Hague, Actes du Colloque de la Société française de radioprotection "Evaluation et surveillance des rejets radioactifs des installations nucléaires », Strasbourg, 13-14 novembre 2002.

Masarik J., Beer J. (1999) Simulation of particle fluxes and cosmogenic nuclide production in the Earth's atmosphere, J. Geophys. Res. 104, 12099-12111.

O'Brien (1979) Secular variations in the production of cosmogenic isotopes in the earth's atmosphere, J. Geophys. Res. 84, 423-431.

Olson P. (2002) The disappearing dipole, Nature 416, 591-594.

Rahmstorf S. (2002) Ocean circulation and climate during the past 120,000 years, Nature 419, 207-214.

Samson T., Siclet F. (2002) EDF et le carbone-14, Actes de la journée carbone-14 de la SFRP (18 avril 2002), Société française de radioprotection, Paris.

Shackleton N.J., Duplessy J.C., Arnold M., Maurice P., Hall M.A., Cartlidge J. (1988) Radiocarbon age of the last glacial deep water, Nature 335, 708-711.

Siegenthaler U., Sarmiento J.L. (1993) Atmospheric carbon dioxide and the ocean, Nature 365, 119 125. 
Sonett C.P., Morfill G.E., Jokipii J.R. (1987) Interstellar shock waves and ${ }^{10}$ Be from ice cores, Nature 330, 458-460.

Stocker T.F., Schmittner A. (1997) Influence of $\mathrm{CO}_{2}$ emission rates on the stability of the thermohaline circulation, Nature 388, 862-865.

Stuiver M., Quay P. (1980) Changes in atmospheric carbon-14 attributed to a variable sun, Science 207, 11-19.

Trumbore S. (2000) Age of soil organic matter and soil respiration: Radiocarbon constraints on belowground C dynamics, Ecolog. Applications 10, 399-411.

Trumbore S., Chadwick O.A., Amundson R. (1996) Rapid exchange between soil carbon and atmospheric carbon dioxide driven by temperature change, Science 272, 393-396.

UNSCEAR (2000) Report of the United Nation Scientific Committee on the Effects of Atomic Radiations to the General Assembly. United Nations, New York.

Voelker A., Sarnthein M., Grootes P.M., Erlenkauser H., Laj C., Mazaud A., Nadeau M.J., Schleicher M. (1998) Correlation of marine ${ }^{14} \mathrm{C}$ ages from the Nordic seas with the GISP2 isotope record: implication for ${ }^{14} \mathrm{C}$ calibration beyond $25 \mathrm{ka}$ BP, Radiocarbon 40, 517-534.

Wolstenholme A., Cook G.T., MacKenzie A.B., Naysmith P., Meadows P.S., McDonald P. (1998) The behavior of Sellafield-derived ${ }^{14} \mathrm{C}$ in the Northeast Irish sea, Radiocarbon 40, 447-458. 